\title{
How inertia and limited potentials affect the timing of sectoral abatements in optimal climate policy
}

\author{
Adrien Vogt-Schilb ${ }^{a \dagger}$, Guy Meunier ${ }^{b}$, Stéphane Hallegatte $^{c, d}$ \\ August 28, 2012 \\ ${ }^{\dagger}$ Corresponding author (vogt@centre-cired.fr) \\ ${ }^{a}$ CIRED, Nogent-sur-Marne, France \\ ${ }^{b}$ INRA-UR1303 ALISS, Ivry-Sur-Seine, France \\ ${ }^{c}$ The World Bank, Sustainable Development Network, Washington D.C., USA \\ ${ }^{d}$ École Nationale de la Météorologie, Météo-France, Toulouse, France
}

\begin{abstract}
This paper investigates the optimal timing of greenhouse gas abatement efforts in a multisectoral model with economic inertia, each sector having a limited abatement potential. It defines economic inertia as the conjunction of technical inertia - a social planner chooses investment on persistent abating activities, as opposed to choosing abatement at each time period independently - and increasing marginal investment costs in abating activities. It shows that in the presence of economic inertia, optimal abatement efforts (in dollars per ton) are bellshaped and trigger a transition toward a low-carbon economy. The authors prove that optimal marginal abatement costs should differ across sectors: they depend on the global carbon price, but also on sector-specific shadow costs of the sectoral abatement potential. The paper discusses the impact of the convexity of abatement investment costs: more rigid sectors are represented with more convex cost functions and should invest more in early abatement. The conclusion is that overlapping mitigation policies should not be discarded based on the argument that they set different marginal costs ("different carbon prices") in different sectors.
\end{abstract}

Keywords: climate change mitigation; sectoral policies; optimal policies; optimal timing; inertia; when-flexibility; how-flexibility; overlapping policies

JEL classification: L98, O21, O25, Q48, Q54, Q58 


\section{Introduction}

Many countries committed to reduce their greenhouse gas (GHG) emissions to limit climate change. The European Union, for instance, pledged to reduce its emissions to $20 \%$ below 1990 levels by 2020 . At the global level, the international community recognizes the objective of limiting the increase in global mean temperature to $2^{\circ} \mathrm{C}$. Translating these objectives into policies is difficult. First, a given temperature objective can be reached with different emissions pathways (this is known as the when-flexibility of the mitigation policies). Second, there are many sectors in which it is possible to reduce GHG, from the use of renewable energy to better building insulation and more efficient cars (we call this the how-flexibility). One question is the optimal distribution of emission reductions and corresponding economic costs - across these options and over time.

Economic theory establishes that the optimal policy is to introduce a price signal corresponding to the pigovian tax and to let the market allocate the burden efficiently across sectors. There may be a controversy on which instrument is more desirable to implement the price signal, notably between a tax or quotas (this question has been studied by a whole branch of the literature since the seminal works of Weitzman (1974)), but it is widely accepted in the literature that the optimal approach is to impose a unique, economy-wide carbon price leading to the same marginal abatement cost in all sectors.

In the presence of multiple market failures or externalities, however, a welfare-maximizing climate policy is more complicated to design (Lipsey and Lancaster, 1956). For instance, many abatement activities use new or emerging technologies that are likely to exhibit learning spillovers. This "twinmarket failure" in the area of eco-innovation (Jaffe et al., 2005) can be addressed by combining R\&D subsidies with a carbon price (Fischer and Newell, 2008; Gerlagh et al., 2009; Grimaud et al., 2011; Acemoglu et al., 2012).

This paper addresses another difficulty in the design of climate policies: the role of economic inertia. Many papers investigating the optimal abatement pathway (i.e. how to benefit from the flexibility on when to abate) work from a fixed baseline scenario, and they assume that abatement actions are decided independently at every period, as a function of their costs and benefits (Nordhaus, 1992). When path dependency is introduced, it is mainly in the form of learning by doing, which makes abatement cost decrease with the cumulative aggregated abatement or cumulative investment in abating capital (van der Zwaan et al., 2002; Manne and Richels, 2004).

But path dependency goes beyond learning by doing. Many authors have reframed climate policy in the context of a transition from carbon-intensive to low-carbon economic patterns. In this context, abatement actions at any given point in time have permanent effects. In particular, there is a large technical inertia embedded in infrastructure patterns (e.g., transport networks, electricity production) and spatial patterns (e.g., low-density vs. high-density cities). Ambitious abatement actions at one point in time would thus reduce emissions over the long term. Several authors have studied how this path dependency impacts the optimal timing of GHG reduction (Grubb et al., 1995; Wigley et al., 1996; Grübler and Messner, 1998; Goulder and Mathai, 2000). ${ }^{1}$

In this paper, we compare the baseline-based and the transition-based approaches. We use a simple multi-sector model to highlight the consequences of considering that abatement actions have a permanent impact on emissions (transition-based approach), instead of an evanescent impact on only one period (baseline-based approach). We call economic inertia the combination of technical inertia - in the form of permanent emission reductions - and decreasing returns of abatement investments at one point in time. When economic inertia is taken into account, the optimal marginal abatement effort - in a dollars-per-ton metric — is not growing over time, like in the classical approach with evanescent abatement actions, but is more concentrated over the shorter term. In this case, bell-shaped abatement efforts support a transition toward low-carbon economic patterns.

We also link the question on when to abate to the question on how, i.e. in which economic sector, to abate. We show that in our framework, the sectoral potentials - the maximum amount of abatement that can be realized in each particular sector - have a strong impact on the optimal timing of abatements at the sector level. In practice, each sector is affected by a social cost of sectoral potentials that needs to be subtracted from the social cost of carbon to assess the optimal amount of abatement that should be undertaken. This effect implies that, at each point in time, the marginal costs of abatement investments should be different across sectors, and that the sectors that will take longer to decarbonize should invest more per abated ton of GHG than the others. We show that this is the case for sectors with large potentials.

\footnotetext{
${ }^{1}$ An up-to-date literature review can be found in Cian and Massimo (2011).
} 
Finally, more inert sectors also take longer to decarbonize, and we show that higher inertia can be modeled through a higher convexity of the investment cost function. To assess precisely optimal sectoral efforts requires information on sectoral costs and potentials, but also on inertia, an information that is not reported separately by existing studies (IPCC, 2007; McKinsey and Company, 2007).

In policy terms, these results suggest that higher-than-average efforts are justified in highpotential and highly inert sectors such as urban planning (Guivarch and Hallegatte, 2011).

In section 2, we briefly introduce the classical approach to the definition of an optimal abatement strategy, with a multi-sector model with evanescent abatement options and sectoral potentials, in the spirit of Nordhaus (1992). In this model, the classical result holds: optimality requires that marginal abatement costs are equal in all sectors. Then, we present in section 3 a slightly different model, where abatement options have a permanent impact on emissions. We show that, in this case, marginal abatement efforts should be larger in sectors that will take longer to reach their full abatement potential. We provide an illustrative example based on IPCC abatement costs in section 4 and investigate the role of convexity on investment costs in section 5. Section 6 concludes.

\section{The classical approach to optimal abatement pathways}

Since the seminal article by Nordhaus (1992), optimal abatement trajectories are frequently calculated assuming that a social planner has to choose when to abate GHG emissions. The planner does so by spending on abatement expenditures that reduce emissions at one given point in time; we refer to these abatement expenditures as evanescent abatements. In this framework, the amount of abatement is decided independently at each period, and the cost at one period does not depend on actions undertaken before. In other words, abatement costs exhibit no path dependency. Abatement at time $t, a(t)$, is done at a cost $\gamma(a(t))$, where $\gamma$ is convex, positive and twice differentiable. These convex costs capture increasing costs in the abatement potentials, i.e. the fact that some emissions are cheaper to abate than others.

In this section, we expand the classical model by describing the economy as a set of sectors indexed by $i$. For simplicity, we assume that these sectors do not interact with each other. The social planner decides the non-negative abatement effort $a_{i}(t)$ done in each sector, using the abatement cost function $\gamma_{i}\left(a_{i}(t)\right)$ which is also convex, positive and twice differentiable: ${ }^{2}$

$$
\begin{aligned}
& \gamma_{i}{ }^{\prime \prime}>0 \\
& \gamma_{i}{ }^{\prime}>0
\end{aligned}
$$

The function $\gamma_{i}{ }^{\prime}\left(a_{i}(t)\right)$ represents the marginal abatement cost (MAC), i.e. the cost of the last unit of abatement at time $t$.

For instance, abatement in the transport sector can be done by changing individual vehicles. Carbon-efficient vehicles typically have higher investment cost but lower operating costs than carbonintensive vehicles. For this reason, intensively-used vehicles may be replaced by zero-carbon vehicles at a lower total cost than those driven occasionally. If vehicles are replaced in the merit order - from the cheapest to the most expensive - the marginal cost of doing so $\gamma_{i}^{\prime}\left(a_{i}(t)\right)$ is growing in $a_{i}(t)$, and $\gamma_{i}\left(a_{i}(t)\right)$ is convex. In economic terms, the convexity on $\gamma_{i}$ comes from zero-carbon vehicles being imperfect substitutes to thermal vehicles.

Moreover, we introduce a sectoral potential $\bar{a}_{i}$ in each sector $i$, which represents the maximum amount of $\mathrm{GHG}$ emissions (in $\mathrm{GtCO}_{2}$ per year) that can be abated in this sector.

$$
\forall i, \forall t, a_{i}(t) \leq \bar{a}_{i}
$$

For instance, if each vehicle is replaced by a zero-emission vehicle, all the abatement potential in the private mobility sector has been realized. The sectoral potential may be roughly approximated by sectoral emissions in the baseline, but they may also be smaller (if there are some fatal emissions in the sector) or could even be higher (if negative emissions are possible).

The climate policy is modeled as a so-called carbon budget for emissions above a given level. We assume that the environment is able to absorb a constant flow of GHG emissions $E_{0} \geq 0$. Above $E_{0}$, emissions are dangerous. The objective is to maintain cumulative dangerous emissions below a given ceiling $B$ (Allen et al., 2009). For simplicity, we assume that emissions would be constant in

\footnotetext{
${ }^{2}$ All notations are reproduced in Tab. 1.
} 


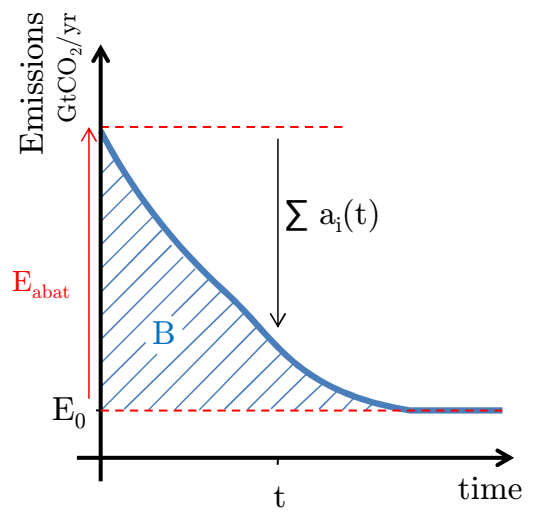

Figure 1: An illustration of the climate constraint. The cumulative emissions above $E_{0}$ should be lower than an intertemporal carbon budget $B$ (this requires that the long-run emissions tend to $E_{0}$ ). Dangerous emissions $E_{\text {abat }}$ are measured from $E_{0}$.

absence of abatement (Fig. 1). We assume that all dangerous emissions are abatable, and, without loss of generality, that doing so requires to use all the abating potential in every sector. Denoting $E_{\text {abat }}$ the emissions above $E_{0}$, this reads:

$$
E_{\text {abat }}=\sum_{i} \bar{a}_{i}
$$

Using another representation of climate policy objectives would not affect the qualitative results of this analysis.

The social planner determines how and when to abate in order to minimize abatement costs discounted at a given rate $r$, under the constraints set by the sectoral potentials and the carbon budget:

$$
\begin{array}{ll} 
& \min _{a_{i}(t)} \int_{0}^{\infty} e^{-r t} \sum_{i} \gamma_{i}\left(a_{i}(t)\right) d t \\
\text { subject to } & a_{i}(t) \leq \bar{a}_{i} \\
& \int_{0}^{\infty}\left(E_{\text {abat }}-\sum_{i} a_{i}(t)\right) d t \leq B
\end{array}
$$

Under these assumptions, the classical result holds: the optimal strategy is to implement abatement options such that marginal abatement costs are equal in all sectors and equal to the current carbon price. With the objective to maintain cumulated emissions below the carbon budget, the (present value of the) social cost of carbon is constant, we denote it $\mu$. As its current value $\mu e^{r t}$ increases at the discounting rate $r$, abatement efforts are increasing over time, and each sector $i$ is progressively decarbonized until it reaches the sectoral potential at a date denoted $T_{i}$. After this date, the sectoral marginal abatement costs remain constant:

$$
\gamma_{i}^{\prime}\left(a_{i}(t)\right)= \begin{cases}e^{r t} \mu & t<T_{i} \\ \gamma_{i}^{\prime}\left(\bar{a}_{i}\right) & t \geq T_{i}\end{cases}
$$

These results (proved in annex A) are a straightforward application of the equimarginal principle: unless the sectoral potentials are binding, optimal marginal efforts are equal, in all sectors, to a unique carbon price.

\section{Introducing economic inertia leads to different marginal costs across sectors}

The classical framework assumes that the amount of abatement is decided (and paid) at each period independently. This is a simplifying assumption. For instance, replacing coal-fired plants with gasfired plants actually reduces annual emissions over a long period of time, while abatement costs 


\begin{tabular}{llc}
\hline Name & Description & $\mathrm{Unit}$ \\
\hline$\mu$ & Social cost of carbon (SCC) & $\$ / \mathrm{tCO}_{2}$ \\
$r$ & Discount rate & $\mathrm{yr}^{-1}$ \\
$a_{i}(t)$ & Current abatement in sector $i$ & $\mathrm{tCO}_{2} / \mathrm{yr}$ \\
$\bar{a}_{i}$ & Sectoral potential in sector $i$ & $\mathrm{tCO}_{2} / \mathrm{yr}$ \\
$\gamma_{i}$ & Abatement cost in sector $i$ & $\$ / \mathrm{yr}$ \\
$\gamma_{i}^{\prime}$ & Marginal abatement cost (MAC) in sector $i$ & $\$ / \mathrm{tCO}_{2}$ \\
$x_{i}(t)$ & Current investment in abating activities in sector $i$ & $\left(\mathrm{tCO}_{2} / \mathrm{yr}\right) / \mathrm{yr}$ \\
$c_{i}$ & Cost of investment in sector $i$ & $\$ / \mathrm{yr}$ \\
$c_{i}^{\prime}$ & Marginal investment cost (MIC) in sector $i$ & $\$ /\left(\mathrm{tCO}_{2} / \mathrm{yr}\right)$ \\
$r c_{i}^{\prime}$ & Annualized MICs in sector $i$ & $\$ / \mathrm{tCO}_{2}$ \\
$\lambda_{i}(t)$ & Social cost of the sectoral potential (SCSP) in sector $i$ & $\$ / \mathrm{tCO}_{2}$ \\
\hline
\end{tabular}

Table 1: Notations and units

are mainly paid when the decision is made. In the building sector, reductions are achieved in part by retrofitting buildings, which has an almost-permanent effect on emissions. Another example is urban development, which impacts emissions on the long term (Strand, 2011; Viguié and Hallegatte, 2012). We call this the technical inertia: abatement at time $t$ cannot be controlled directly; instead, it depends on the efforts that have been made before $t$. This introduces path dependency in the analysis.

In our new model, abatement in each sector $i$ starts at zero, and at each time step $t$, the social planner chooses a non-negative amount of physical investment in abating capacities $x_{i}(t)$. Doing so increments the current abatement $a_{i}$ :

$$
\begin{aligned}
a_{i}(0) & =0 \\
\dot{a}_{i}(t) & =x_{i}(t)
\end{aligned}
$$

Where dotted variables represent temporal derivatives. These abatement investments are realized at a cost $c_{i}\left(x_{i}\right)$, the functions $c_{i}$ are twice differentiable, positive, increasing and convex:

$$
\begin{aligned}
c_{i}^{\prime}(0) & =0 \\
c_{i}{ }^{\prime \prime} & >0
\end{aligned}
$$

The values $c_{i}{ }^{\prime}\left(x_{i}\right)$ are the Marginal Investment Cost (MIC) in abating activities, i.e. the cost of the last unit of abatement investment.

The convexity bears on the investments flow, to capture the fact that additional abatements are obtained at an increasing marginal cost (or decreasing returns) in any given sector. For instance, $x_{i}(t)$ could stand for the pace - measured in buildings per year - at which old buildings are being retrofitted at date $t$. The abatement $a_{i}(t)$ would be proportional to the share of retrofitted buildings in the stock. In a given time lapse, retrofitting buildings requires to pay scarce skilled workers. If workers are hired in the merit order and paid at the marginal productivity, the marginal price of retrofitting buildings $c_{i}^{\prime}\left(x_{i}\right)$ is a growing function of $x_{i}$ - in other words, $c_{i}$ is convex. In addition, the existing literature on inertia (Ha-Duong et al., 1997; Lecocq et al., 1998; Schwoon and Tol, 2006) claims that existing studies on limited capital stock turnover justifies this cost convexity: if emitting capital is replaced by cleaner capital slower than the natural turnover rate, marginal costs are constant; if it is replaced faster costs rise nonlinearly.

This convexity is of different nature than the convexity in $\gamma_{i}\left(a_{i}(t)\right)$ in the classical approach presented in section 2, where convexity arises from heterogeneity in abatement options (e.g., different abatement costs for frequently-driven and occasionally-driven vehicles).

We call economic inertia the combination of technical inertia (6) and convex investment costs (8).

Proposition 1 Consider an optimal abating strategy in multiple sectors subject to economic inertia and limited potentials. In general, at each point in time, Marginal Investment Costs (MICs) should not be equal across sectors.

In each sector, MICs should be equal to the social cost of the abated carbon minus the value of the forgone option to abate later. 


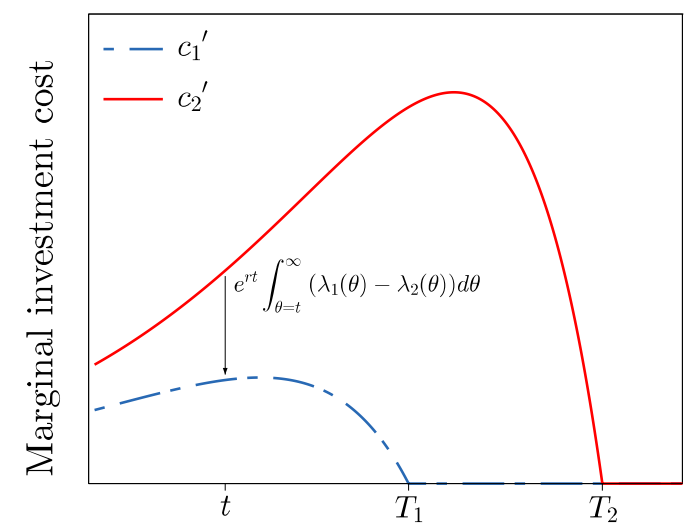

Figure 2: Optimal marginal investment costs (MIC) in abating activities in a case with two sectors $(i \in$ $\{1,2\})$. MICs differ across sectors, because of the social costs of the sectoral potentials. The optimal timing of sectoral abatements comes from a trade-off between investing later in order to reduce present costs thanks to the discounting and investing sooner to benefit from the persistence of the abating efforts over time, resulting in a bell-shaped pathway (16).

Let us sketch the proof to provide some intuitions of the mechanisms at stake. (The full proof is in annex B). The social planner program reads:

$$
\min _{x_{i}(t)} \int_{0}^{\infty} e^{-r t} \sum_{i} c_{i}\left(x_{i}(t)\right) d t
$$

subject to $a_{i}(t) \leq \bar{a}_{i}$

$$
\begin{aligned}
& \int_{0}^{\infty}\left(E_{\text {abat }}-\sum_{i} a_{i}(t)\right) d t \leq B \\
& \dot{a}_{i}(t)=x_{i}(t)
\end{aligned}
$$

The associated Lagrangian reads (see annex B):

$$
\begin{aligned}
L\left(x_{i}, a_{i}, \lambda_{i}, \nu_{i}, \mu\right)= & \int_{0}^{\infty} e^{-r t} \sum_{i} c_{i}\left(x_{i}(t)\right) d t+\int_{0}^{\infty} \sum_{i} \lambda_{i}(t)\left(a_{i}(t)-\bar{a}_{i}\right) d t \\
& +\mu\left(\int_{0}^{\infty}\left(E_{a b a t}-\sum_{i} a_{i}(t)\right) d t-B\right) \\
& -\int_{0}^{\infty} \sum_{i} \dot{\nu}_{i}(t) a_{i}(t) d t-\int_{0}^{\infty} \sum_{i} \nu_{i}(t) x_{i}(t) d t
\end{aligned}
$$

in which $\lambda_{i}, \mu$ and $\nu_{i}$ are the Lagrange multipliers associated with the constraints of the sectoral potential (10), the carbon budget (11) and the abatement dynamic (6) respectively. In particular, the present value of the carbon budget constraint is constant and equal to $\mu$, we call it the social cost of carbon (SCC); $\mu e^{r t}$ is its current value, we call it the (current) carbon price.

First-order conditions read:

$$
\begin{aligned}
& \frac{\partial L}{\partial a_{i}}=0 \Longleftrightarrow \dot{\nu}_{i}(t)=\lambda_{i}(t)-\mu \\
& \frac{\partial L}{\partial x_{i}}=0 \Longleftrightarrow c_{i}{ }^{\prime}\left(x_{i}(t)\right)=e^{r t} \nu_{i}(t)
\end{aligned}
$$

The optimal marginal investment $\operatorname{costs} c_{i}\left(x_{i}(t)\right)$ in sector $i$ depends on the unique SCC $\mu$ and on the sector-specific social costs of the sectoral potential (SCSP) $\lambda_{i}$ (see details in annex B). The social planner controls $x_{i}$ but their integral $a_{i}$ is bounded by the potentials $\bar{a}_{i}$, making these potentials affect the optimal strategy before they are reached:

$$
c_{i}^{\prime}\left(x_{i}(t)\right)=e^{r t} \int_{t}^{\infty}\left(\mu-\lambda_{i}(\theta)\right) d \theta
$$


The left hand side of (15) is the cost of the last unit of investment in abating capacity, i.e. the Marginal Investment Cost (MIC), in sector $i$. The integral in the right hand side is different across sectors. It represents the total current value $\left(e^{r t}\right)$ of the carbon that will be saved from $t$ onwards thanks to the marginal investment, minus the sector-specific total SCSP from $t$ onwards. The SCSP $\lambda_{i}(t)$ is null before the sectoral potential has been reached. Once the potential has been reached, the option to abate more carbon in sector $i$ is removed $\left(\lambda_{i}(\theta)=\mu\right)$.

Noting $T_{i}$ the dates when the sectoral potentials are reached, ${ }^{3}$ we can express explicitly the optimal marginal investment costs in each sector as a function of the optimal SCC $\mu$ and the optimal dates $T_{i}$ (see annex B):

$$
c_{i}{ }^{\prime}\left(x_{i}(t)\right)= \begin{cases}\mu e^{r t}\left(T_{i}-t\right) & \text { if } t<T_{i} \\ 0 & \text { if } t \geq T_{i}\end{cases}
$$

Marginal investments should be priced at the social cost of the carbon that they will save until the sectoral potential is reached. The longer it takes for a sector to reach its potential (i.e. the larger $T_{i}$ ), the higher should be priced investments in abating capacity.

In particular, marginal investment costs are not equal to the current carbon price $\mu e^{r t}$, and not equal across sectors.

The optimal investments pathways $x_{i}(t)$ can be found by inverting (16). They arise from a complex trade-off: investing soon allows the planner to benefit from the persistence of abatements, and prevents to invest too much in the long-term; but it brings closer the date $T_{i}$, removing the option to invest later, when the discount factor is higher. This results in a bell-shaped distribution of mitigation costs over time (Fig. 2): in the short term, the effect of discounting dominates and efforts grow exponentially; in the long term, the effect of the limited potential dominates and the effort decrease to zero.

A corollary of (15) is that differences in MICs come from different social costs of the sectoral potentials (Fig. 2):

$$
c_{i}{ }^{\prime}\left(x_{i}(t)\right)-c_{j}{ }^{\prime}\left(x_{j}(t)\right)=e^{r t} \int_{t}^{\infty} \lambda_{j}(\theta)-\lambda_{i}(\theta) d \theta
$$

In particular, optimal strategies may imply paying for expensive abatement while cheaper ones are available:

Corollary 1 Assume that abatement in two sectors $\{1,2\}$ exhibit the same cost structure, and that the first one has a greater potential than the second:

$$
\begin{aligned}
\forall x>0, \quad c_{1}(x) & =c_{2}(x) \\
\bar{a}_{1} & <\bar{a}_{2}
\end{aligned}
$$

The second sector will take longer to decarbonize:

$$
T_{1}<T_{2}
$$

At any time, the sector with the largest potential should invest more (both in dollars per abated ton of GHG and in physical terms) than the other.

$$
\forall t, \quad c_{1}^{\prime}\left(x_{1}(t)\right)<c_{2}^{\prime}\left(x_{2}(t)\right) \quad \text { and } \quad x_{1}(t)<x_{2}(t)
$$

The proof is in annex C. Even if the cost function is the same in both sectors, different sectoral potentials cause the investment along the optimal path to be different across sectors. This result is explained by the combination of limited sectoral potentials and increasing marginal investments costs - without convex investment costs, the sectors would be decarbonized in the merit order: the cheapest sector first.

\footnotetext{
${ }^{3}$ After $T_{i}, x_{i}(t)=0$ and $c_{i}{ }^{\prime}\left(x_{i}(t)\right)=0$.
} 


\begin{tabular}{|c|c|c|}
\hline & Marginal cost & Abatement potential \\
\hline & $\gamma_{i}^{\prime}\left(1 \mathrm{GtCO}_{2} / \mathrm{yr}\right) \quad\left[\$ / \mathrm{tCO}_{2}\right]$ & $\bar{a}_{i} \quad\left[\mathrm{GtCO}_{2} / \mathrm{yr}\right]$ \\
\hline Waste & 34 & 0.76 \\
\hline Industry & 17.6 & 4.08 \\
\hline Forestry & 15.9 & 2.75 \\
\hline Agriculture & 11.9 & 4.39 \\
\hline Transport & 11.6 & 2.1 \\
\hline Energy & 10.3 & 3.68 \\
\hline Buildings & 3.6 & 5.99 \\
\hline
\end{tabular}

Table 2: Assumptions on marginal abatement costs and maximum potential in seven sectors of the global economy. Numerical values derived from the abatement potential at a marginal cost below $20 \$ / \mathrm{tCO}_{2}$ in figure SPM.6, page 11 of IPCC (2007).

\section{Illustrative examples using IPCC abatement costs}

We use the two models (with and without economic inertia) to investigate the optimal sectoral abatements over the 2007-2030 period. We set a policy objective over this period only, ${ }^{4}$ and use abatement cost information derived from the IPCC (2007). Because of data limitations, this exercise is not supposed to suggest an optimal climate policy (see also section 5). It aims at illustrating the impact of two extreme assumptions (abatement options are totally evanescent or totally permanent) on the optimal abatement strategy, using the same cost estimates and the same policy objective. We show that the optimal abatement strategy - and in particular the choice of which sectors need to abate more and earlier — depends on whether abatement actions are assumed permanent or evanescent.

\subsection{Data}

IPCC (2007) provides data on abatement costs and potentials until 2030, in 7 sectors of the global economy (Tab. 2). These abatement costs have two components that are aggregated in IPCC data. The first component is the cost for evanescent reduction; it is captured by the model exposed in section 2. The other component is the costs of persistent reductions, it can be captured by the model exposed in section 3. From the same data on costs, potentials and carbon budget, we derive two optimal reduction strategies, one from the model with no economic inertia (section 2), and the other from the model with technical inertia and convex investment costs (section 3).

In this illustrative example, we assume abatable emissions $E_{a b a t}=\sum_{i} \bar{a}_{i}=24 \mathrm{GtCO}_{2} / \mathrm{yr}$. Compared to the total baseline emissions in 2030 in the A1B scenario from Nakicenovic et al. (2000), $69 \mathrm{GtCO}_{2} / \mathrm{yr}$, this gives $E_{0}=45 \mathrm{GtCO}_{2} / \mathrm{yr}$. In this case, $E_{0}$ cannot be interpreted as a definitive safe level for emissions, but rather as an emission milestone in 2030 in a pathway toward a sustainable longer-term target.

We call $T=23$ yr the time span from 2007 - the publication date of IPCC (2007) — and 2030. We set the discount rate to $r=4 \% / \mathrm{yr}$. We constrain the cumulative dangerous emissions over the period as:

$$
\int_{0}^{T}\left(E_{\text {abat }}-\sum_{i} a_{i}(t)\right) \leq 140 \mathrm{GtCO}_{2}
$$

The two models are solved with a numerical resolution, using a time step $d t=0.1 \mathrm{yr}^{5}$

\subsection{Calibration}

We calibrate the two models (with and without inertia) such that the discounted costs of reaching the same target are equal in the two models (this methodology was first used by Grubb et al. (1995)).

\footnotetext{
4 The theoretical, infinite-horizon models exposed in sections 2 and 3 have to be modified; all the results exposed still apply.

${ }^{5}$ Data and source code are available at the corresponding author's web page. Computation and plots use Scilab (Scilab Consortium, 2011).
} 


\begin{tabular}{|c|c|c|c|c|}
\hline & \multicolumn{2}{|c|}{ MAC parameter } & \multicolumn{2}{|c|}{ MIC parameter } \\
\hline & $\gamma_{i}^{m}$ & {$\left[\frac{\$ / \mathrm{tCO}_{2}}{\mathrm{GtCO}_{2} / y r}\right]$} & $c_{i}^{m}$ & {$\left[\frac{\$ / \mathrm{tCO}_{2}}{\mathrm{GtCO}_{2} / \mathrm{yr}^{3}}\right.$} \\
\hline Waste & & 34 & & 3732 \\
\hline Industry & & 17.6 & & 1932 \\
\hline Forestry & & 15.9 & & 1745 \\
\hline Agriculture & & 11.9 & & 1306 \\
\hline Transport & & 11.6 & & 1273 \\
\hline Energy & & 10.3 & & 1131 \\
\hline Buildings & & 3.6 & & 395.2 \\
\hline
\end{tabular}

Table 3: Calibration of the Marginal Investment Cost (MIC) functions and of the Marginal Abatement Costs (MAC) functions. This figures are consistent with our modeling framework $(18,20)$ and the data from IPCC reported in the Tab. 2.

We also ensure that relative costs (when comparing two sectors) are equal in the two models. This way, differences in optimal strategies arise only from the introduction of economic inertia.

We first solve the problem exposed in section 2, assuming quadratic abatement costs. Quadratic costs are a simple specification that grants that the $\gamma_{i}$ are convex, and simplifies the resolution as marginal abatement costs are linear:

$$
\begin{aligned}
\forall i, \quad \forall x \geq 0, \quad \gamma_{i}(a) & =\frac{\gamma_{i}^{m}}{2} a^{2} \\
\forall x \geq 0, \quad \gamma_{i}{ }^{\prime}(a) & =\gamma_{i}^{m} \cdot a
\end{aligned}
$$

Where $\gamma_{i}^{m}$ are parameters specific to each sector. We calibrate these costs functions using the abatements corresponding to a $20 \$ / \mathrm{tCO}_{2}$ marginal cost in figure SPM.6 of IPCC (2007) presented in Tab. 2; the result of this calibration is presented in the first column of Tab. 3.

The numerical resolution allows one to compute the optimal strategy in financial $\left(\gamma_{i}^{\prime}\left(a_{i}(t)\right)\right)$ and physical $\left(a_{i}(t)\right)$ terms (see (34) and (36) in annex D and Fig. 3).

We call $\Gamma$ the discounted cost of the optimal mitigation strategy:

$$
\Gamma=\int_{0}^{T} e^{-r t} \sum_{i} \gamma_{i}\left(a_{i}(t)\right) d t
$$

The numerical resolution gives $\Gamma=3351 \mathrm{G} \$$.

Then, we solve the model with economic inertia, still assuming quadratic costs.

$$
\forall i, \quad \forall x \geq 0, \quad c_{i}(x)=\frac{c_{i}^{m}}{2} x^{2}
$$

This allows one to simplify the model (see (38) and (41) in annex E). We calibrate the $c_{i}^{m}$ parameters by adapting the methodology from Grubb et al. (1995): first, we assume that the relative MICs are equal to the relative MACs from IPCC:

$$
\forall i, \quad \frac{c_{i}^{m}}{c_{1}^{m}}=\frac{\gamma_{i}^{m}}{\gamma_{1}^{m}}
$$

And second, we constrain the total costs of the mitigation strategy to be equal in the two models:

$$
\sum_{i} \int_{0}^{\infty} e^{-r t} c_{i}\left(x_{i}(t)\right) d t=\Gamma
$$

A numerical resolution provides $c_{i}^{m}$ (Tab. 3), as well as the optimal abating strategy $x_{i}$ and $a_{i}$ (Fig. 3)

Finally, we introduce annualized marginal investment costs. In our modeling framework, evanescent options are implemented paying a MAC, while permanent options are implemented paying a MIC. Investing $x_{i}(t)$ at $t$ reduces GHG emissions from $t$ onwards, but the cost of doing so, $c_{i}\left(x_{i}(t)\right)$, is paid only at time $t$. To make this cost easier to compare with evanescent abatement costs, it is useful to introduce the annualized cost of investment $r \cdot c_{i}\left(x_{i}(t)\right)$ and its marginal value. Indeed, paying the 
amount $c_{i}{ }^{\prime}\left(x_{i}(t)\right)$ at time $t$ is equivalent to paying the flow $r \cdot c_{i}{ }^{\prime}\left(x_{i}(t)\right)$ from $t$ to $\infty$. Multiplying all terms in equation (16) by $r$, one gets:

$$
r \cdot c_{i}{ }^{\prime}\left(x_{i}(t)\right)= \begin{cases}r\left(T_{i}-t\right) \mu e^{r t} & \text { if } t<T_{i} \\ 0 & \text { if } t \geq T_{i}\end{cases}
$$

Annualized MICs are expressed in $\$ / \mathrm{tCO}_{2}$ so they can be compared easily to the marginal abatement costs derived from the model without inertia (Fig. 3). Even if they can be expressed in the same metrics, annualized MICs differ from the MACs à la Nordhaus (1992): as explained in sections 2 and 3, MICs depend on the decarbonizing pace in each sector, while MAC only depend on the current abatement (also in each sector).

\subsection{Results}

The optimal mitigation strategy by the two models are compared sector by sector in Fig. 3, and the aggregated pathways in terms of abatement and financial effort are presented in Fig. 4. The pathways suggested by these models are not optimal pathways to mitigate climate change: they only consider a target in the 2007-2030 window and are based on crude available data.

The two models give the same result in the long run: abatement in each sector eventually reaches its maximum potential. By construction, they also achieve aggregated abatement target at the same discounted cost. But the optimal strategies according to the two models differ radically in terms of aggregated abatement and financial costs, and, more importantly, at the sector level.

The optimal abatement pathway in the model without economic inertia includes significant abatements as soon as the climate policy is implemented, while the model with inertia has a more realistic abatement pathway that starts at zero and increases progressively over time.

In terms of financial effort, the model with inertia provides an optimal pathway that starts higher and decreases over time toward zero - this is the decreasing side of the bell-shaped trajectory discussed in the formal analysis (Fig. 2). In this case, abatement efforts are transitional, once all the capital in a sector has been replaced by non-emiting capital, financial efforts are not required anymore. While the carbon price grows exponentially in both model (see the discussion after equation (12)), the aggregated financial efforts tend to zero when economic inertia is taken into account. This is consistent with proposition 1: in this case, marginal investement costs in abating activities should not be equal to the carbon price.

Taking into account economic inertia also modifies the optimal sectoral burden sharing. Without inertia, all sectors are decarbonized up to the same marginal effort until they reach their sectoral potential. When inertia is taken into account, the optimal timing of sectoral reductions arises from a complex trade-off. Investing soon in abating activities allows the planner to benefit from the persitency of abatements; but it brings closer the date when the sectoral potential is reached, removing the option to invest later, when the discounted cost is lower.

To avoid paying too much at any point in time, investment should be spread over a larger period, and longer-to-decarbonize sectors should abate at a higher cost than the others. In this example with quadratic investment costs - i.e. with the same cost convexity accross sectors —, industry is decarbonized faster (in terms of $\left.x_{i}(t)\right)$ and at a higher cost $\left(r \cdot c_{i}{ }^{\prime}\left(x_{i}(t)\right)\right)$ than forestry, despite forestry being a priori cheaper to decarbonize. This is because industry has a greater abatement potential (Tab. 2) and thus takes longer to decarbonize.

These results are not policy recommendations, as we considered only a limited time period. Moreover, the assumption of a uniform convexity accross sectors is inappropriate; we used it in absence of data on sectoral inertia. The next section discusses the effect of different cost convexities on economic inertia and optimal economic pathways.

\section{The impact of cost convexity on optimal abatement strategies}

The classical model (without economic inertia) suggests that buildings should be entirely decarbonized by 2011, being the first sector to reach its full abatement potential (bottom right panel of Fig. 3). In the baseline-based modeling framework, this result is natural, as the model implements abating activities in the merit order (4). As available studies find that better insulation in the buildings would largely pay for themselves thanks to energy savings, resulting in very low, or even negative abatement costs (McKinsey and Company, 2007), this model will always recommend to decarbonize buildings very fast. 

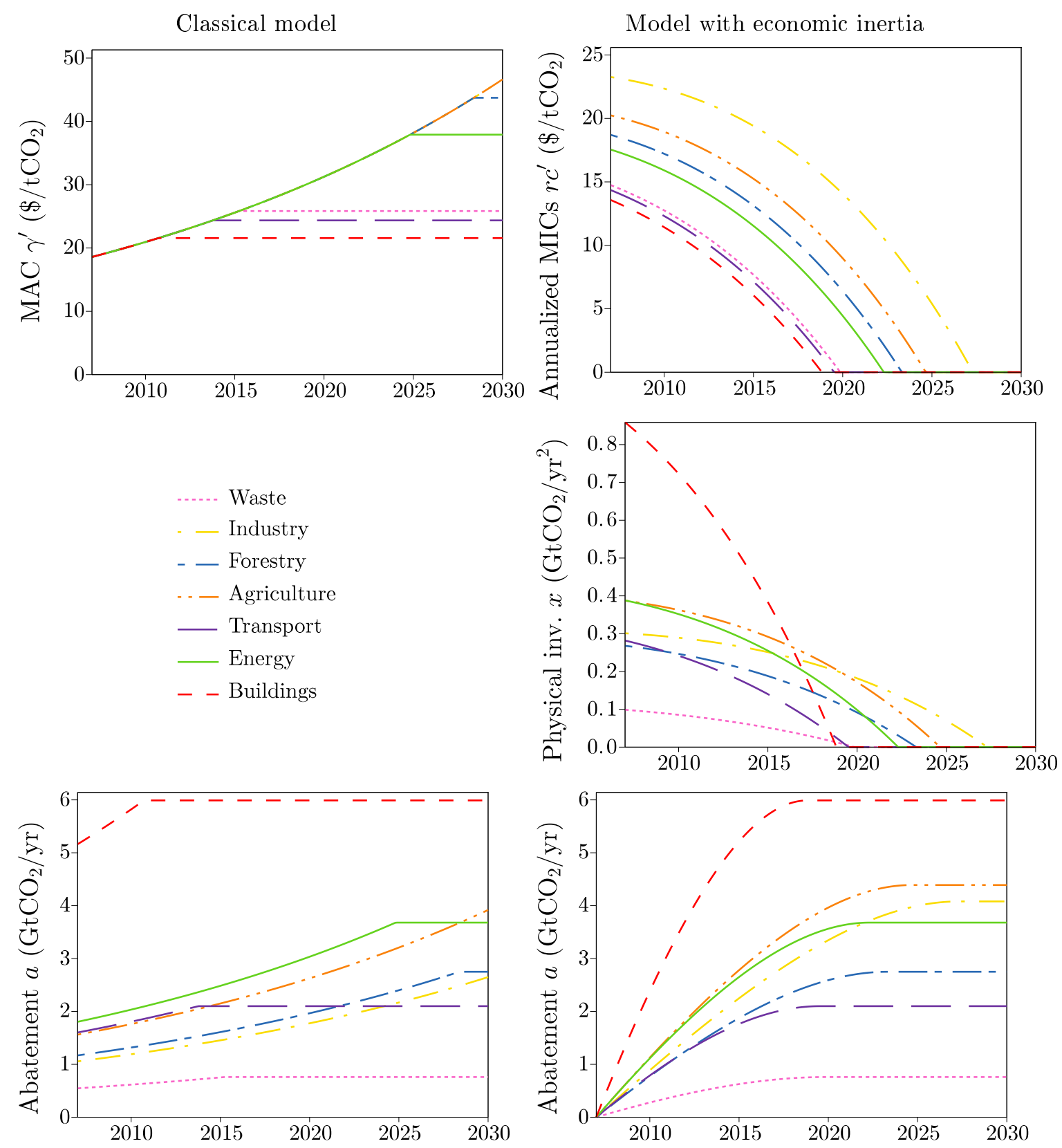

Figure 3: Comparison of marginal costs and optimal abatement strategies to achieve the same amount of abatement, when the costs from table 2 are understood as marginal abatement costs (left) vs. annualized marginal investment costs (right).

In the first case, without economic inertia, sectoral efforts should be equal to the current carbon price, unless they have reached the maximum potential (upper left panel). As a consequence, the abatement may jump from 0 to a non-negative value instantaneously at the beginning of the period (as for instance in the buildings sector, lower left panel).

In the right column, with economic inertia, the abatement starts at zero (lower panel) and increases progressively with the accumulation of physical investments (middle). In this case, abatement in longerto-decarbonize sectors should be priced higher than abatement in quick-to-decarbonize ones (upper panel). Note: in the classical model, there is no equivalent to the physical investments in abating activities $x_{i}$, as the planner controls directly the abatement level $a_{i}$ without having to deal with economic inertia. 
This is not satisfying: it will arguably take decades to retrofit all existing energy-consuming buildings at the reported cost. Previous research has tackled this issue by imposing exogenous upper bounds in the decarbonizing pace of any sector (Vogt-Schilb and Hallegatte, 2011). In our model with economic inertia, mitigation costs depend on how fast the sector is decarbonized. We demonstrate below that in this model, different convexities bearing on the investment cost function allow to take into account different inertias.

We start from the model with economic inertia and linear MICs, but, this time, we choose a particular sector $b=$ Buildings for which we use a more general cost function (for all other sectors, we keep the same quadratic cost functions than before), allowing to investigate the influence of cost convexity on the results:

$$
\forall x \geq 0, \quad c_{b}(x)=\frac{1}{\alpha_{b}}\left(\frac{x}{x_{b}^{r e f}}\right)^{\alpha_{b}}
$$

Where $\alpha_{b}>1$ describes the convexity of the costs function. In economic terms, $\alpha_{b}$ is the constant cost elasticity of abating investments in sector $b$ :

$$
\frac{d c_{b}}{d x_{b}} \cdot \frac{c_{b}}{x_{b}}=\alpha_{b}
$$

Finally, $x_{b}^{r e f}$ (in the same unit than $x_{b}$ ) is a scale parameter. For each value of $\alpha_{b}$, we calibrate $x_{b}^{r e f}\left(\alpha_{b}\right)$ such that the total mitigation cost of the building sector remains unchanged. ${ }^{6}$

We investigate the impact of $\alpha_{b}$ on the optimal timing of the mitigation strategy. First, we compare the optimal strategies in a reference case (with $\alpha_{b}=2$, replicating the case of quadratic costs functions) and a case with higher convexity $\alpha_{b}=2.3$. The corresponding optimal abatement pathways are shown in Fig. 5. Compared to the reference case, the building sector takes much more time to decarbonize when its costs are more convex: the date $T_{b}$ moves from 2016 to 2030 . To respect the carbon budget, the social planner must abate faster in all others sectors, which is achieved with a higher carbon cost (not shown).

We then perform a full sensitivity analysis, solving the same model for a range of posible $\alpha_{b}$ (Fig. 6). When $\alpha_{b}$ tends to 1 , the MIC $c_{b}^{\prime}$ does not depend on the decarbonizing pace $x_{b}$ anymore, and there is no inertia. The building sector is fully decarbonized instantaneously at the beginning of the period. On the other hand, the greater $\alpha_{b}$ the longer the building sector takes to reach its abating potential.

These results confirm that different economic inertias can be modeled through different convexities of investment costs in a model with persistent abatements. In a given sector, the optimal timing strongly depends on the convexity of its investments cost function. In this example, when $\alpha_{b}$ varies from 1.9 to $2.1, T_{b}$ varies from 2013 to 2021; in the first case, decarbonizing buildings take 6 years, in the latter 14 , more than twice more. ${ }^{7}$

These results also show that the question of how and when to abate cannot be answered based on costs and potential information alone. In addition, information on inertia is required. VogtSchilb and Hallegatte (2011) suggest to expand existing marginal abatement cost curves — existing MACCs currently report costs and potentials of a set of abatement options - with information on the implementation pace of each option. This paper confirms this need in an analytical framework.

\section{Discussion and conclusion}

In this paper, we modify a classical model à $l a$ Nordhaus (1992) and introduce economic inertia bearing on GHG mitigation activities. We define economic inertia as the conjunction of technical inertia (a social planner chooses investment on persistent abating activities, as opposed to choosing abatement at each time step independently) and increasing marginal investment costs. We investigate the optimal timing of abatement efforts in a multisectoral model that accounts for economic inertia in abating activities, and in which each sector has a limited abatement potential. We compare the

\footnotetext{
${ }^{6}$ We could have decided to keep $x_{b}^{r e f}$ constant instead, without modifying the qualitative results and the message from the experiment. But this would not have granted that investment costs are held constant except in the particular case when $x_{b}=x_{b}^{r e f}$.

${ }^{7} T_{b}$ is less sensible to $\alpha_{b}$ if we hold $x_{b}^{\text {ref }}$ constant instead of the total cost of decarbonizing buildings (see also footnote 6).
} 

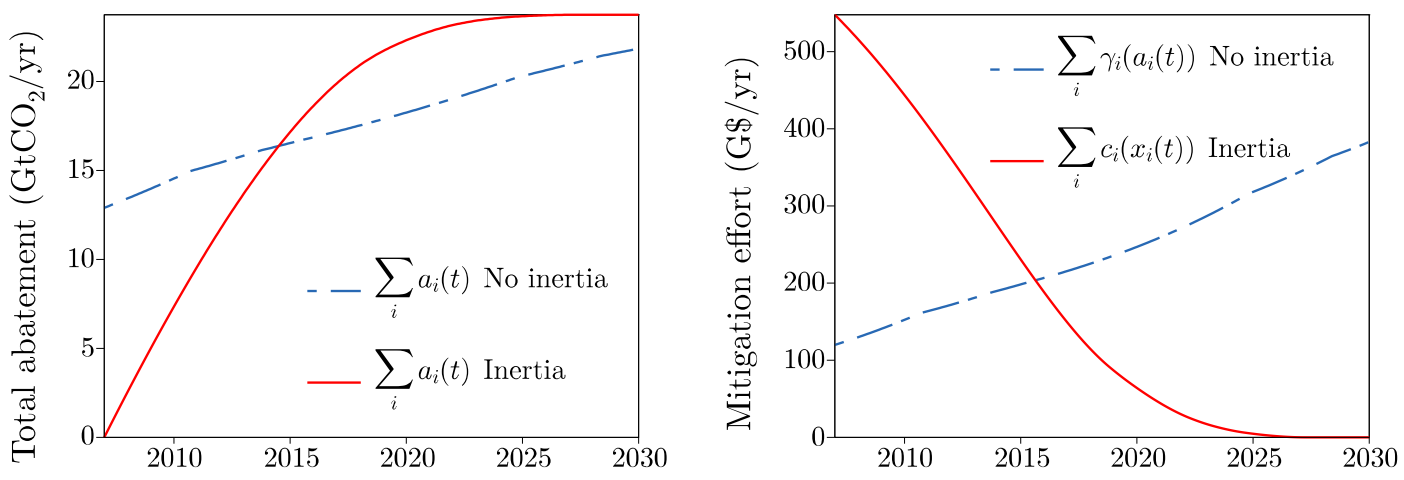

Figure 4: Comparison of financial and physical efforts in the models with inertia vs. without inertia. When economic inertia is disregarded, the abatement can jump from 0 to a non-negative value instantaneously at the beginning of the period. When economic inertia is taken into account, abatement has to grow continuously, and short-term financial efforts are higher than when inertia is neglected. By construction, the total discounted cost and the cumulative abatements are equal in the two cases.

Low convexity $\alpha_{b}=2$

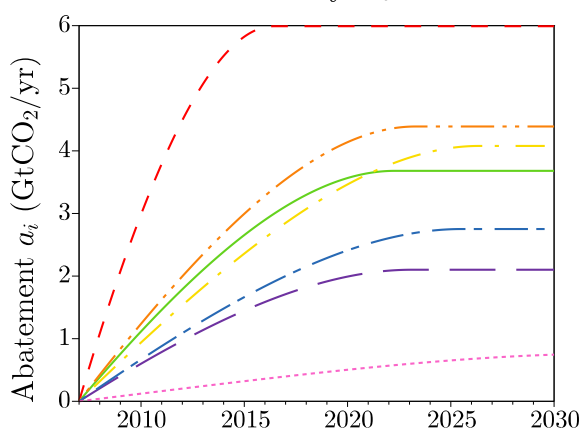

High convexity $\alpha_{b}=2.3$

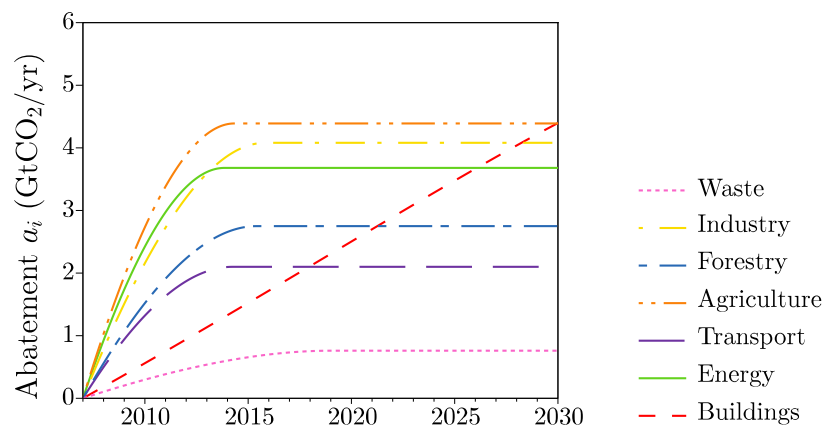

Figure 5: Increasing the convexity of the building sector cost function has a direct and an indirect effect. The direct is that it increases economic inertia of the building sectors and spreads its abatement over the period (even if total abatement cost in the building sector is held constant). The indirect is that abatement in the other sectors must be achieved faster to reach the carbon budget.

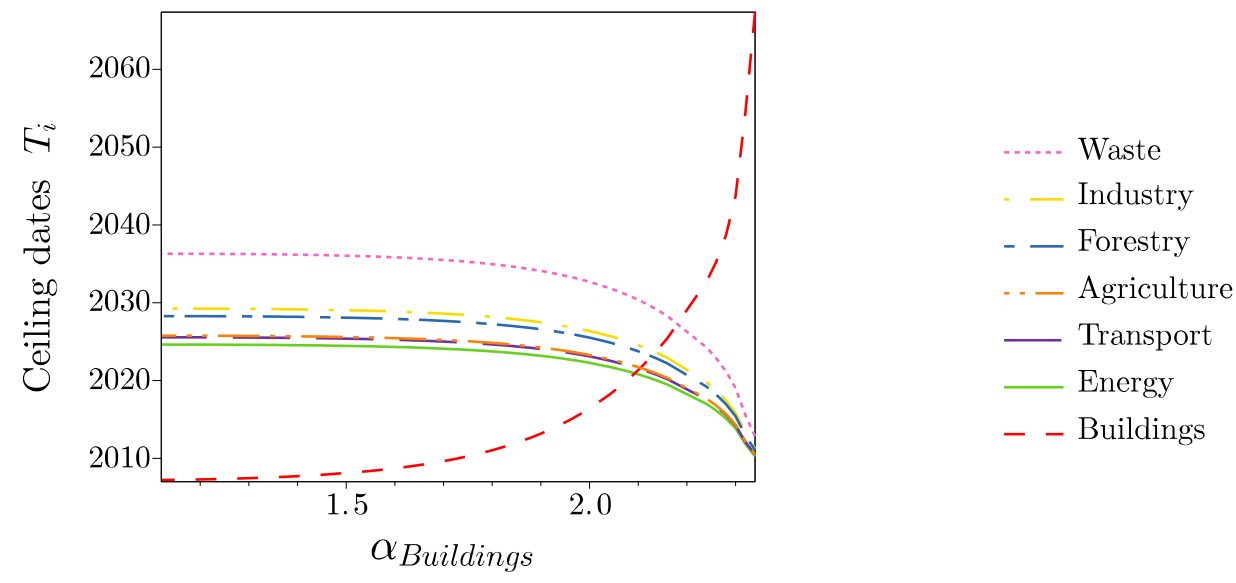

Figure 6: Optimal decarbonizing dates as a function of $\alpha_{\text {Buildings. }}$ The more the cost function of the building sector is convex, the more progressive should be the abatement in this sector: $T_{\text {Buildings }}$ is postponed. Moreover, the other sectors have to decarbonize faster to cope with the global carbon budget. 
results obtained with our new model and those coming from a classical model without economic inertia.

These two models illustrate the difference between two approaches to the climate change problem. Initially, most analyses considered a given GHG emission (and development) baseline and abatement expenditures applied to this unchanged baseline. In such a setting, abatement expenditures need to increase over time roughly at the discount rate, and they need to do so for ever. This is what is reproduced by our model with no inertia, a simplified version of the seminal model by Nordhaus (1992). In another approach, it has been proposed to consider the climate change issue as a development pathway problem (World Bank, 2010, 2012). In such a framework, what is needed is a bifurcation toward a different, low-carbon development model. In this case, abatement efforts are seen as transitional, they are required to shift from carbon-intensive to low-carbon economic patterns.

Here, we link the question on when to abate to the question on how, i.e. in which sector, to abate. The two models have diverging recommendation at the sector level. The model with no inertia suggests that all sectors should implement abatement options up to a given marginal abatement cost, equal for all sectors. This leads to unrealistic recommendations at the sector level (e.g., retrofit all old buildings by 2011). On the opposite, the model with economic inertia suggests that sectors with higher potential (e.g., industry) should invest more than other sectors (e.g., forestry) per abated ton at every point in time. Marginal investment costs in abating activities should be priced to a global carbon price less a sector-specific social cost of the sectoral potential.

The difference between the two models illustrates that the design of an optimal abatement pathway requires reporting separately abatement costs arising from, on the one hand, imperfect substitution between high- and low-emitting capital, and, on the other hand, transitional costs. Costs from the second category depend on the decarbonizing pace, strongly impacting the optimal time profile of an abating strategy.

The model with economic inertia shows that optimal marginal efforts in a given sector depend positively on the time it will take to reach the sectoral abatement potential. In inert sectors, investment in abating activities should be priced higher than average, and could require specific policies such as norms and regulations (e.g., feed-in tariffs, efficiency standards or land-use planning). This result provides an analytical framework to support previous findings that larger efforts are desirable in sectors with high inertia and large potential (Jaccard and Rivers, 2007; Shalizi and Lecocq, 2009; Vogt-Schilb and Hallegatte, 2011).

There is currently a debate on the overlapping mitigation policies, as the $3 \times 20$ objectives of the European Union, existing standards on automobile, buildings and household appliance, and feed-in tariffs for specific low carbon technologies in the power sector. Such overlapping policies have been criticized, partly because they set different marginal costs in different economic sectors. Previous research has shown that differences in marginal abatement costs can be justified, notably by learning spillovers and other market imperfections (Braathen, 2007; Fischer and Preonas, 2010). We bring a new argument based on the existence of limited sectoral potentials and economic inertia: investments in abating activities should be priced at the cost of the carbon they allow to abate until the sectoral potential is reached; in sectors that will take long to decarbonize, higher effort levels are justified.

\section{Acknowledgments}

The authors wish to thank Patrice Dumas, Marianne Fay, Frédérique Ghersi, Louis-Gaëtan Giraudet, Céline Guivarch, Oskar Lecuyer, Baptiste Perissin Fabert, Antonin Pottier, Julie Rozenberg and Ankur Shah Delight for useful comments. We also thank Patrice Dumas for technical support. The views expressed in this paper are the sole responsibility of the authors. They do not necessarily reflect the views of the World Bank, its executive directors, or the countries they represent.

\section{References}

Acemoglu, D., Aghion, P., Bursztyn, L., Hemous, D., 2012. The environment and directed technical change. American Economic Review 102 (1), 131-166.

Allen, M. R., Frame, D. J., Huntingford, C., Jones, C. D., Lowe, J. A., Meinshausen, M., Meinshausen, N., 2009. Warming caused by cumulative carbon emissions towards the trillionth tonne. Nature 458 (7242), 1163-1166. 
Braathen, N. A., 2007. Instrument mixes for environmental policy: How many stones should be used to kill a bird? International Review of Environmental and Resource Economics 1 (2), 185-235.

Cian, E., Massimo, T., 2011. Mitigation portfolio and policy instruments when hedging against climate policy and technology uncertainty. Environmental Modeling \& Assessment 17 (1-2), 123136.

Fischer, C., Newell, R. G., 2008. Environmental and technology policies for climate mitigation. Journal of Environmental Economics and Management 55 (2), 142-162.

Fischer, C., Preonas, L., 2010. Combining policies for renewable energy: Is the whole less than the sum of its parts? International Review of Environmental and Resource Economics 4, 51-92.

Gerlagh, R., Kverndokk, S., Rosendahl, K. E., 2009. Optimal timing of climate change policy: Interaction between carbon taxes and innovation externalities. Environmental and Resource Economics 43 (3), 369-390.

Goulder, L. H., Mathai, K., 2000. Optimal CO2 abatement in the presence of induced technological change. Journal of Environmental Economics and Management 39 (1), 1-38.

Grübler, A., Messner, S., 1998. Technological change and the timing of mitigation measures. Energy Economics 20 (5-6), 495-512.

Grimaud, A., Lafforgue, G., Magné, B., 2011. Climate change mitigation options and directed technical change: A decentralized equilibrium analysis. Resource and Energy Economics 33 (4), 938-962.

Grubb, M., Chapuis, T., Ha-Duong, M., 1995. The economics of changing course : Implications of adaptability and inertia for optimal climate policy. Energy Policy 23 (4-5), 417-431.

Guivarch, C., Hallegatte, S., 2011. Existing infrastructure and the $2{ }^{\circ} \mathrm{C}$ target. Climatic Change 109 (3-4), 801-805.

Ha-Duong, M., Grubb, M. J., Hourcade, J. C., 1997. Influence of socioeconomic inertia and uncertainty on optimal CO 2-emission abatement. Nature 390, 271.

IPCC, 2007. Summary for policymakers. In: Climate change 2007: Mitigation. Contribution of working group III to the fourth assessment report of the intergovernmental panel on climate change. Cambridge University Press, Cambridge, UK and New York, USA.

Jaccard, M., Rivers, N., 2007. Heterogeneous capital stocks and the optimal timing for CO2 abatement. Resource and Energy Economics 29 (1), 1-16.

Jaffe, A. B., Newell, R. G., Stavins, R. N., 2005. A tale of two market failures: Technology and environmental policy. Ecological Economics 54 (2-3), 164-174.

Lecocq, F., Hourcade, J., Ha-Duong, M., 1998. Decision making under uncertainty and inertia constraints: sectoral implications of the when flexibility. Energy Economics 20 (5-6), 539-555.

Lipsey, R. G., Lancaster, K., 1956. The general theory of second best. The Review of Economic Studies $24(1), 11-32$

Manne, A., Richels, R., 2004. The impact of learning-by-doing on the timing and costs of CO2 abatement. Energy Economics 26 (4), 603-619.

McKinsey, Company, 2007. Reducing US greenhouse gas emissions: How much at what cost? Tech. rep., McKinsey \& Co.

Nakicenovic, N., Alcamo, J., Davis, G., de Vries, B., Fenhann, J., Gaffin, S., Gregory, K., Grubler, A., Jung, T. Y., Kram, T., et al., 2000. Special report on emissions scenarios: a special report of working group III of the intergovernmental panel on climate change. Tech. rep., Pacific Northwest National Laboratory, Richland, WA (US), Environmental Molecular Sciences Laboratory (US).

Nordhaus, W. D., 1992. An optimal transition path for controlling greenhouse gases. Science 258 (5086), 1315-1319. 
Schwoon, M., Tol, R. S., 2006. Optimal CO2-abatement with socio-economic inertia and induced technological change. The Energy Journal 27 (4).

Scilab Consortium, 2011. Scilab: The free software for numerical computation. Scilab Consortium, Paris, France.

Shalizi, Z., Lecocq, F., 2009. Climate change and the economics of targeted mitigation in sectors with Long-Lived capital stock. World Bank Policy Research Working Paper 5063.

Strand, J., 2011. Inertia in infrastructure development: Some analytical aspects, and reasons for inefficient infrastructure choices. Journal of Infrastructure Development 2 (1), 51-70.

van der Zwaan, B. C. C., Gerlagh, R., G., Klaassen, Schrattenholzer, L., 2002. Endogenous technological change in climate change modelling. Energy Economics 24 (1), 1-19.

Viguié, V., Hallegatte, S., 2012. Trade-offs and synergies in urban climate policies. Nature Climate Change 2 (5), 334-337.

Vogt-Schilb, A., Hallegatte, S., 2011. When starting with the most expensive option makes sense: Use and misuse of marginal abatement cost curves. Policy Research Working Paper 5803, World Bank, Washington DC, USA.

Weitzman, M. L., 1974. Prices vs. quantities. The Review of Economic Studies 41 (4), 477-491.

Wigley, T. M. L., Richels, R., Edmonds, J. A., 1996. Economic and environmental choices in the stabilization of atmospheric CO2 concentrations. Nature 379 (6562), 240-243.

World Bank, 2010. Development and climate change. World Bank, Washington.

World Bank, 2012. Inclusive green growth : the pathway to sustainable development. World Bank, Washington, D.C. 


\section{A Proof of (4)}

The associated Lagrangian reads:

$$
\begin{aligned}
L\left(a_{i}, \lambda_{i}, \mu\right)= & \int_{0}^{\infty} e^{-r t} \sum_{i} \gamma_{i}\left(a_{i}(t)\right) d t+\int_{0}^{\infty} \sum_{i} \lambda_{i}(t)\left(a_{i}(t)-\bar{a}_{i}\right) d t \\
& +\mu\left(\int_{0}^{\infty}\left(E_{\text {abat }}-\sum_{i} a_{i}(t)\right) d t-B\right)
\end{aligned}
$$

The first order condition is:

$$
\begin{aligned}
\frac{\partial L}{\partial a_{i}}=0 & \Longleftrightarrow e^{-r t} \gamma_{i}^{\prime}\left(a_{i}(t)\right)+\lambda_{i}(t)-\mu=0 \\
& \Longleftrightarrow \gamma_{i}^{\prime}\left(a_{i}(t)\right)=e^{r t}\left(\mu-\lambda_{i}(t)\right)
\end{aligned}
$$

Let $T_{i}$ be the date when the abatement potential $\bar{a}_{i}$ is reached in sector $i$, such that:

$$
\begin{aligned}
& \forall t<T_{i}, a_{i}(t)<\bar{a}_{i} \\
& \forall t \geq T_{i}, a_{i}(t)=\bar{a}_{i}
\end{aligned}
$$

As the associated Lagrangian multiplier, $\lambda_{i}(t)$ is null before the sectoral potential becomes binding:

$$
\begin{aligned}
\forall t & \lambda_{i}(t)\left(a_{i}(t)-\bar{a}_{i}\right)=0 \\
\Longrightarrow \forall t<T_{i}, & \lambda_{i}(t)=0
\end{aligned}
$$

Combining (27) and (26), one gets:

$$
\gamma_{i}^{\prime}\left(a_{i}(t)\right)= \begin{cases}e^{r t} \mu & t<T_{i} \\ \gamma_{i}^{\prime}\left(\bar{a}_{i}\right) & t \geq T_{i}\end{cases}
$$

This is the classical result: in every sector, current marginal abatement costs should be equal to the current carbon price $\left(e^{r t} \mu\right)$, unless the sectoral potential has been exhausted already.

\section{B Full proof of the proposition 1}

The associated Lagrangian reads:

$$
\begin{aligned}
L\left(x_{i}, a_{i}, \dot{a}_{i}, \lambda_{i}, \nu_{i}, \mu\right)= & \int_{0}^{\infty} e^{-r t} \sum_{i} c_{i}\left(x_{i}(t)\right) d t+\int_{0}^{\infty} \sum_{i} \lambda_{i}(t)\left(a_{i}(t)-\bar{a}_{i}\right) d t \\
& +\mu\left(\int_{0}^{\infty}\left(E_{\text {abat }}-\sum_{i} a_{i}(t)\right) d t-B\right)+\int_{0}^{\infty} \sum_{i} \nu_{i}(t)\left(\dot{a}_{i}(t)-x_{i}(t)\right) d t
\end{aligned}
$$

In the last term, $\dot{a}_{i}(t)$ can be removed thanks to an integration by parts:

$$
\begin{aligned}
\int_{t}^{\infty} \sum_{i} \nu_{i}(t) & \left(\dot{a}_{i}(t)-x_{i}(t)\right) d t \\
= & \sum_{i}\left(\int_{0}^{\infty} \nu_{i}(t) \dot{a}_{i}(t) d t-\int_{0}^{\infty} \nu_{i}(t) x_{i}(t) d t\right) \\
& =\sum_{i}\left(\text { constant }-\int_{0}^{\infty} \dot{\nu}_{i}(t) a_{i}(t) d t-\int_{0}^{\infty} \nu_{i}(t) x_{i}(t) d t\right)
\end{aligned}
$$

The transformed Lagrangian does not depend on $\dot{a}_{i}(t)$ :

$$
\begin{aligned}
L\left(x_{i}, a_{i}, \lambda_{i}, \nu_{i}, \mu\right)=\int_{0}^{\infty} & e^{-r t} \sum_{i} c_{i}\left(x_{i}(t)\right) d t+\int_{0}^{\infty} \sum_{i} \lambda_{i}(t)\left(a_{i}(t)-\bar{a}_{i}\right) d t \\
& +\mu\left(\int_{0}^{\infty}\left(E_{a b a t}-\sum_{i} a_{i}(t)\right) d t-B\right) \\
& -\int_{0}^{\infty} \sum_{i} \dot{\nu}_{i}(t) a_{i}(t) d t-\int_{0}^{\infty} \sum_{i} \nu_{i}(t) x_{i}(t) d t
\end{aligned}
$$


The first order conditions read:

$$
\begin{aligned}
& \frac{\partial L}{\partial a_{i}}=0 \Longleftrightarrow \dot{\nu}_{i}(t)=\lambda_{i}(t)-\mu \\
& \frac{\partial L}{\partial x_{i}}=0 \Longleftrightarrow e^{-r t} c_{i}{ }^{\prime}\left(x_{i}(t)\right)=\nu_{i}(t)
\end{aligned}
$$

The abatement $a_{i}(t)$ are increasing over time $\left(\right.$ as $\left.\dot{a}_{i}(t)=x_{i}(t) \geq 0\right)$. For each sector $i$ there is a date $T_{i}$ such that

$$
\begin{aligned}
& \forall t<T_{i}, a_{i}(t)<\bar{a}_{i} \quad \Longrightarrow \lambda_{i}(t)=0 \\
& \forall t \geq T_{i}, a_{i}(t)=\bar{a}_{i} \quad \Longrightarrow \lambda_{i}(t) \geq 0
\end{aligned}
$$

The Lagrange multipliers $\lambda_{i}(t)$ are null before $T_{i}$ and strictly positive after. So, by integrating (13):

$$
\begin{aligned}
\nu_{i}(t) & =\nu_{i}\left(T_{i}\right)+\int_{T_{i}}^{t} \dot{\nu}_{i}(t) \\
\forall t<T_{i}, \nu_{i}(t) & =\nu_{i}\left(T_{i}\right)+\left(T_{i}-t\right) \mu
\end{aligned}
$$

Furthermore, for $t>T_{i}$ the abatement $a_{i}(t)$ is constant, equal to $\bar{a}_{i}$, so $x_{i}(t)=\dot{a}_{i}(t)$ is null, and, from (14) and (7):

$$
\forall t \geq T_{i}, \nu_{i}(t)=0
$$

Therefore,

$$
\nu_{i}(t)= \begin{cases}\left(T_{i}-t\right) \mu & \text { if } t<T_{i} \\ 0 & \text { if } t \geq T_{i}\end{cases}
$$

Then, from (14), the marginal costs can be expressed as a function of $T_{i}$ :

$$
c_{i}{ }^{\prime}\left(x_{i}(t)\right)= \begin{cases}e^{r t}\left(T_{i}-t\right) \mu & \text { if } t<T_{i} \\ 0 & \text { if } t \geq T_{i}\end{cases}
$$

Marginal investment costs should be equal to the total social cost of the carbon $(\mu)$ that will be saved thanks to the abatement before the sectoral potential is reached (the time span $\left(T_{i}-t\right)$ ), as expressed in current value $\left(e^{r t}\right)$.

\section{Proof of corollary 1}

Proof: As $c_{i}{ }^{\prime}$ is by assumption (7) strictly growing, it is invertible. Let $\chi_{i}$ be the inverse of $c_{i}{ }^{\prime}$ :

$$
\chi_{i}=c_{i}{ }^{\prime-1}
$$

Applying $\chi_{i}$ to (16) gives:

$$
x_{i}(t)= \begin{cases}\chi_{i}\left(e^{r t}\left(T_{i}-t\right) \mu\right) & \text { if } t<T_{i} \\ 0 & \text { if } t \geq T_{i}\end{cases}
$$

The relation between the sectoral potential $\left(\bar{a}_{i}\right)$, the MICs (through $\left.\chi_{i}\right)$, the SCC $(\mu)$ and the time it takes to achieve the sectoral potential $T_{i}$ reads:

$$
\begin{aligned}
\bar{a}_{i} & =a_{i}\left(T_{i}\right) \\
& =\int_{0}^{T_{i}} \chi_{i}\left(e^{r t}\left(T_{i}-t\right) \mu\right) d t
\end{aligned}
$$

Let us define $f_{\chi_{i}}$ such that:

$$
\begin{aligned}
f_{\chi_{i}}(t) & =\int_{0}^{t} \chi_{i}\left(e^{r \theta}(t-\theta) \mu\right) d \theta \\
\Longrightarrow \frac{d f_{\chi_{i}}}{d t}(t) & =\int_{0}^{t} e^{r \theta} \chi_{i}{ }^{\prime}\left(e^{r \theta}(t-\theta) \mu\right) d \theta
\end{aligned}
$$


Let us show that $f_{\chi_{i}}$ is invertible: $\chi_{i}{ }^{\prime}>0$ as the inverse of $c^{\prime}>0$, thus $\frac{d f_{\chi_{i}}}{d t}>0$ and therefore $f_{\chi_{i}}$ is strictly growing. Finally:

$$
\bar{a}_{i} \mapsto T_{i}=f_{\chi_{i}}{ }^{-1}\left(\bar{a}_{i}\right) \text { is an increasing function }
$$

For a given marginal cost function, ${ }^{8} T_{i}$ can always be found from $\bar{a}_{i}$. The larger the potential, the longer it takes for the optimal strategy to achieve it.

Noting that MICs in sector $i$ depend increasingly on $T_{i}$ (16) finishes the demonstration.

\section{Details on the linear MAC model}

Let us define the inverted marginal abatement cost functions $\phi_{i}$ :

$$
\forall q \geq 0, \quad \phi_{i}(q)=\frac{q}{\gamma_{i}^{m}}
$$

They allow calculating the optimal strategy by inverting (4):

$$
a_{i}(t)= \begin{cases}\phi_{i}\left(e^{r t} \mu\right) & t<T_{i} \\ \bar{a}_{i} & t \geq T_{i}\end{cases}
$$

The relationship between the sectoral potential, the carbon price and the decarbonization date is:

$$
\bar{a}_{i}=\frac{\mu}{\gamma_{i}^{m}} e^{r T_{i}}
$$

And then, the emissions from sector $i$ are:

$$
E_{i}=\int_{0}^{T_{i}} \bar{a}_{i}-a_{i}(t) d t=\int_{0}^{T_{i}}\left[e^{r T_{i}}-e^{r t}\right] \frac{\mu}{\gamma_{i}^{m}} d t=\frac{\mu}{r \gamma_{i}^{m}}\left[T_{i} e^{r T_{i}}+e^{r T_{i}}-1\right]
$$

This allows one to express the carbon constraint in function of the decarbonization dates:

$$
\begin{aligned}
& B=\sum_{i} E_{i} \\
& B=\sum_{i} \frac{\mu}{r \gamma_{i}^{m}}\left(T_{i} e^{r T_{i}}+e^{r T_{i}}-1\right)
\end{aligned}
$$

We then calculate the total mitigation costs $\Gamma_{i}$ in the sector $i$ before date $T$ given SCC $\mu$.

$$
\begin{aligned}
\Gamma_{i} & =\int_{0}^{T} e^{-r t} \gamma_{i}\left(a_{i}(t)\right) d t \\
& =\int_{0}^{T_{i}} e^{-r t} \frac{\gamma_{i}^{m}}{2}\left(\frac{e^{r t} \mu}{\gamma_{i}^{m}}\right)^{2} d t+\frac{\gamma_{i}^{m}}{2} r \bar{a}_{i}^{2} \frac{1}{r}\left[e^{-r T_{i}}-e^{-r T}\right]
\end{aligned}
$$

injecting this expression of $\bar{a}_{i}(34$ and 33$)$ :

$$
\Gamma_{i}=\frac{\mu^{2}}{2 \gamma_{i}^{m}} \frac{1}{r}\left[e^{r T_{i}}-1\right]+\frac{\mu^{2}}{2 \gamma_{i}^{m}} \frac{1}{r}\left[e^{r T_{i}}-e^{r\left(2 T_{i}-T\right)}\right]
$$

then

$$
\Gamma_{i}=\frac{\mu^{2}}{2 r \gamma_{i}^{m}}\left[2 e^{r T_{i}}-1-e^{r\left(2 T_{i}-T\right)}\right]
$$

\footnotetext{
${ }^{8}$ When $c_{i}{ }^{\prime}$ is given, $\chi_{i}$ and therefore $f_{\chi_{i}}$ are also given.
} 


\section{E Details on the linear MIC model}

Investment costs are quadratic:

$$
\begin{aligned}
\forall i, \quad \forall x \geq 0, \quad c_{i}(x) & =\frac{c_{i}^{m}}{2} x^{2} \\
\forall x \geq 0, \quad c_{i}{ }^{\prime}(x) & =c_{i}^{m} \cdot x
\end{aligned}
$$

Let us define the inverted marginal investment cost functions $\chi_{i}=c_{i}^{\prime-1}$ :

$$
\forall p \geq 0, \quad \chi_{i}(p)=\frac{p}{c_{i}^{m}}
$$

With this specification, it is possible to write the optimal investment strategy $x_{i}$ :

$$
x_{i}(t)=\frac{e^{r t}\left(T_{i}-t\right) \mu}{c_{i}^{m}}
$$

then, to link the date $T_{i}$ to the sectoral potential and the cost of carbon $\mu$ :

$$
\bar{a}_{i}=\frac{\mu}{c_{i}^{m} r^{2}}\left(e^{r T_{i}}-1-r T_{i}\right)
$$

The emissions beyond $E_{0}$ from sector $i$ are:

$$
\begin{aligned}
E_{i} & =\int_{0}^{T_{i}} \bar{a}_{i}-a_{i}(t) d t \\
& =\int_{0}^{T_{i}}\left[\int_{0}^{T_{i}} x_{i}(\theta) d \theta-\int_{0}^{t} x_{i}(\theta) d \theta\right] d t \\
& =\int_{t=0}^{T_{i}}\left[\int_{\theta=t}^{T_{i}} x_{i}(\theta) d \theta\right] d t=\int_{\theta=0}^{T_{i}} \int_{t=0}^{\theta} x_{i}(\theta) d t d \theta \\
& =\int_{0}^{T_{i}} \theta x_{i}(\theta) d \theta \\
& =\frac{\mu}{c_{i}^{m}} \int_{0}^{T_{i}} \theta\left(T_{i}-\theta\right) e^{r \theta} d \theta \\
& =\frac{\mu}{c_{i}^{m} r^{3}}\left[r T_{i}\left(e^{r T_{i}}+1\right)-2\left(e^{r T_{i}}-1\right)\right]
\end{aligned}
$$

And the carbon budget satisfies:

$$
\begin{aligned}
B & =\sum_{i} E_{i} \\
B & =\sum_{i} \frac{\mu}{c_{i}^{m}} \frac{1}{r^{3}}\left[r T_{i}\left(e^{r T_{i}}+1\right)-2\left(e^{r T_{i}}-1\right)\right]
\end{aligned}
$$

We calculate the total mitigation $\operatorname{costs} C_{i}$ in sector $i$ :

$$
\begin{aligned}
C_{i} & =\int_{0}^{\infty} e^{-r t} c_{i}\left(x_{i}(t)\right) d t \\
& =\int_{0}^{T_{i}} e^{-r t} \frac{c_{i}^{m}}{2}\left(\frac{e^{r t}\left(T_{i}-t\right) \mu}{c_{i}^{m}}\right)^{2} d t \\
& =\frac{\mu^{2}}{2 c_{i}^{m}} \int_{0}^{T_{i}} e^{r t}\left(T_{i}-t\right)^{2} d t \\
C_{i} & =\frac{\mu^{2}}{c_{i}^{m} r^{3}}\left(e^{r \cdot T_{i}}-1-r \cdot T_{i}-\frac{1}{2}\left(r \cdot T_{i}\right)^{2}\right)
\end{aligned}
$$

